\title{
Optogenetic control of the BMP signalling pathway
}

Paul A. Humphreys ${ }^{1,2}$, Steven Woods ${ }^{1}$, Christopher A. Smith1, Nicola Bates ${ }^{1}$, Stuart A. Cain ${ }^{1}$, Robert Lucas ${ }^{2} \&$ Susan J. Kimber ${ }^{*}$

${ }^{1}$ Division of Cell Matrix \& Regenerative Medicine, Faculty of Biology, Medicine and Health, The University of Manchester, Manchester, M13 9PL, UK

2Division of Neuroscience \& Experimental Psychology, Faculty of Biology, Medicine and Health, The University of Manchester, Manchester, M13 9PL, UK

*Corresponding Author: Prof Susan J. Kimber. Email: susan.kimber@manchester.ac.uk 


\section{List of Supporting Information}

Figure S1: Vector maps of viral shuttle plasmids

Figure S2: Single cell quantification of nuclear P-SMAD1/5 fluorescence in optoBMP-TC28a2 cells

Figure S3: Analysis of SMAD2 nuclear translocation in optoBMP-TC28a2 cells

Figure S4: Activation of non-canonical signalling after blue light stimulation in optoBMPTC28a2 cells

Figure S5: Gene expression analyses of BMP-like receptors in optoBMP-TC28a2 cells. 
A

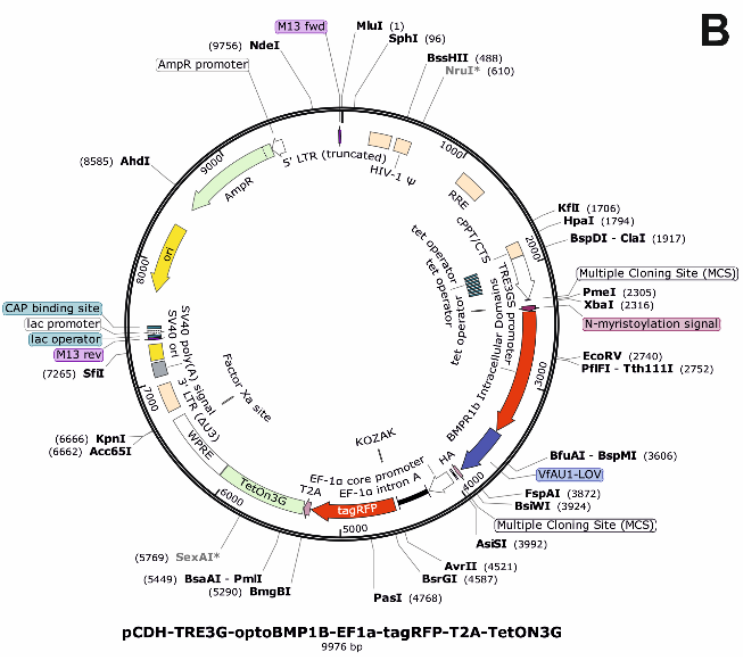

C

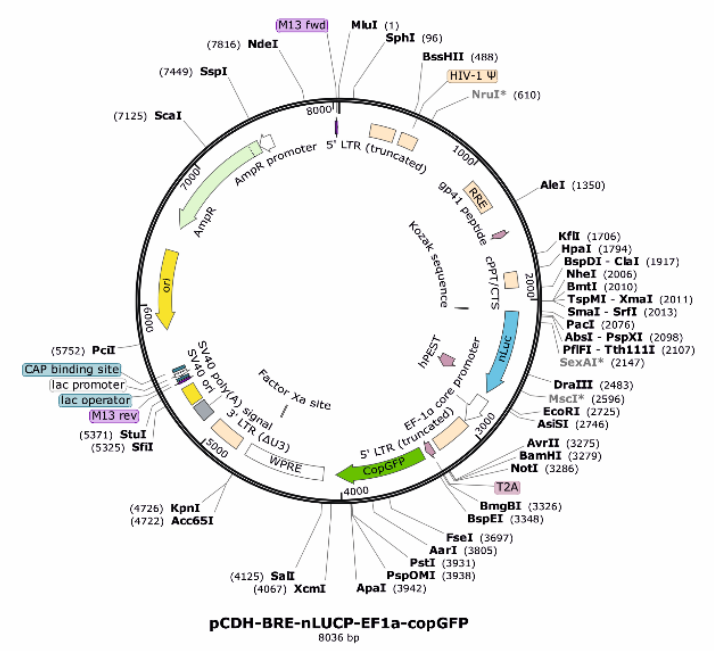

B

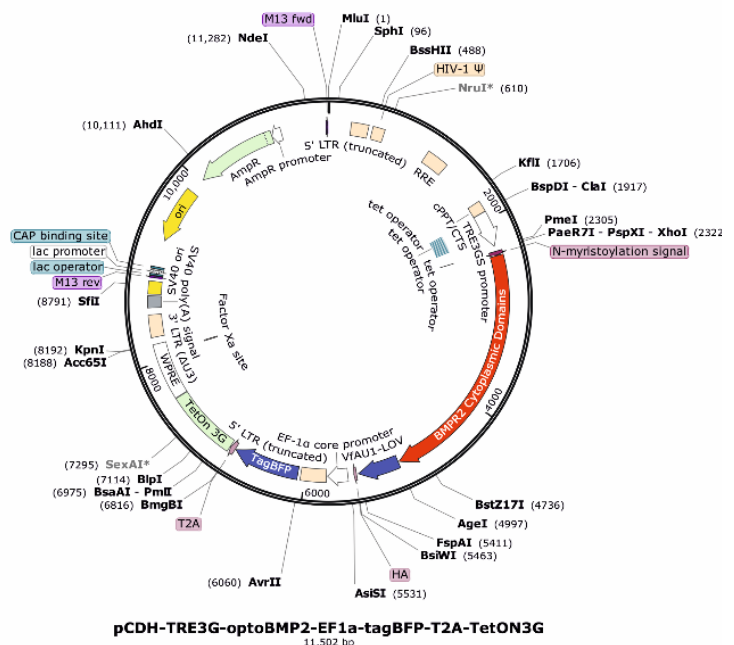

pCDH-TRE3G-optoBMP2-EF 1a-tagBFP-T2A-TetoN3G

Figure S1 - Vector maps.
(A) Full vector map of TetOn-optoBMPR1B
(B) Full vector map of TetOn-optoBMPR2
(C) Full vector map of pCDH-BRE-nLUCP-EF1a-copGFP 
A

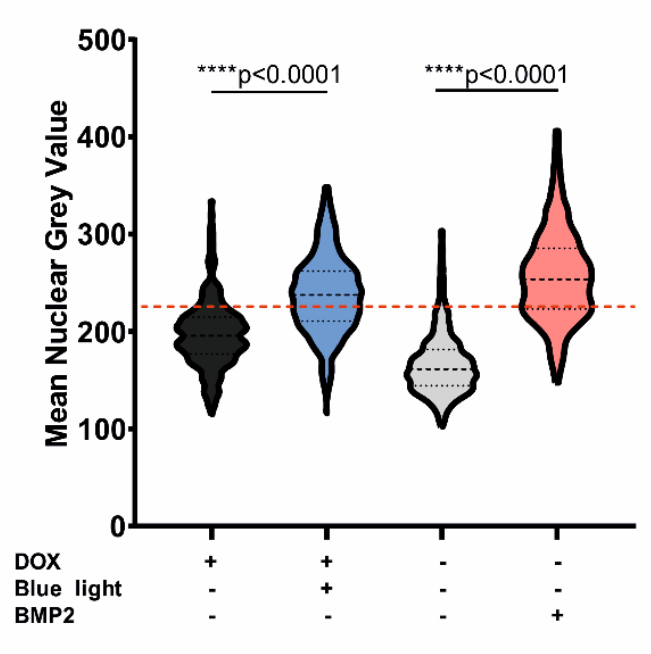

B

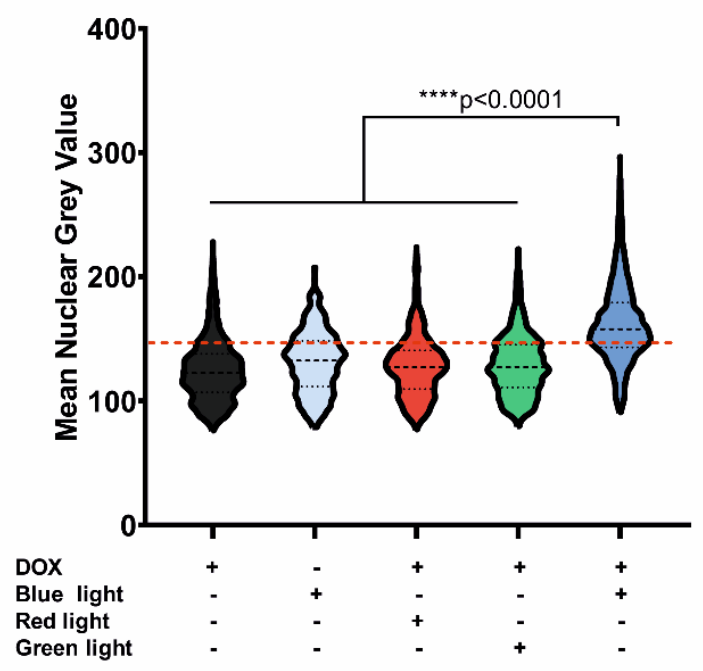

Figure S2. Single cell quantification of nuclear P-SMAD1/5 fluorescence. (A-B) Quantification of nuclear P-SMAD1/5 mean grey value when (A) cells were stimulated with blue light $0 \mathrm{f} 5 \mathrm{ng} / \mathrm{ml}$ BMP2 and (B) stimulated with a range of light wavelengths. $N=$ three different fields of view per condition across three independent experiments.

Data Information: Nuclei were identified with DAPI channel and relative nuclear fluoresence intensity of P-SMAD1/5 was measured with ImageJ. Quantification performed using three different fields of view per condition across three independent experiments. Violin plots represent all quantified data. Dotted red line indicates mean grey value threshold for positivity determined through analysis of nonstimulated controls. $P$ values were generated using an ordinary one-way ANOVA $\left({ }^{* * * *} p<0.0001\right)$. 
A

DARK
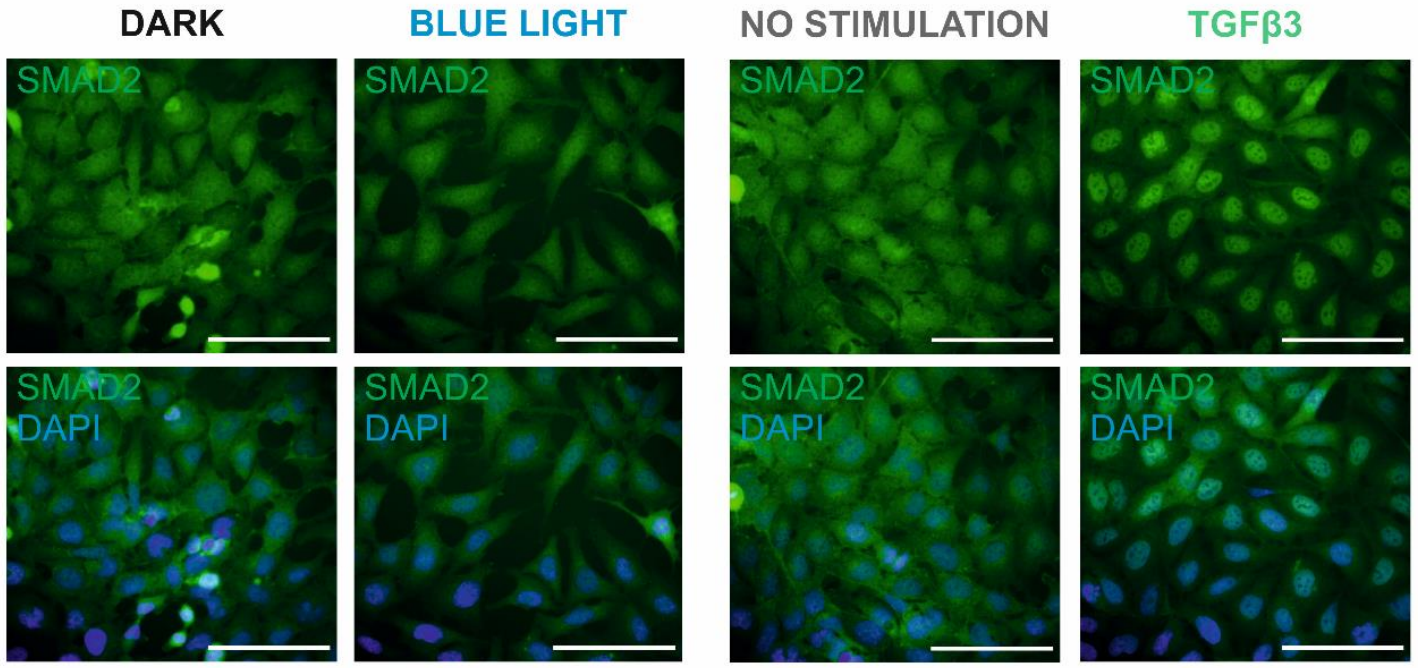

B

C
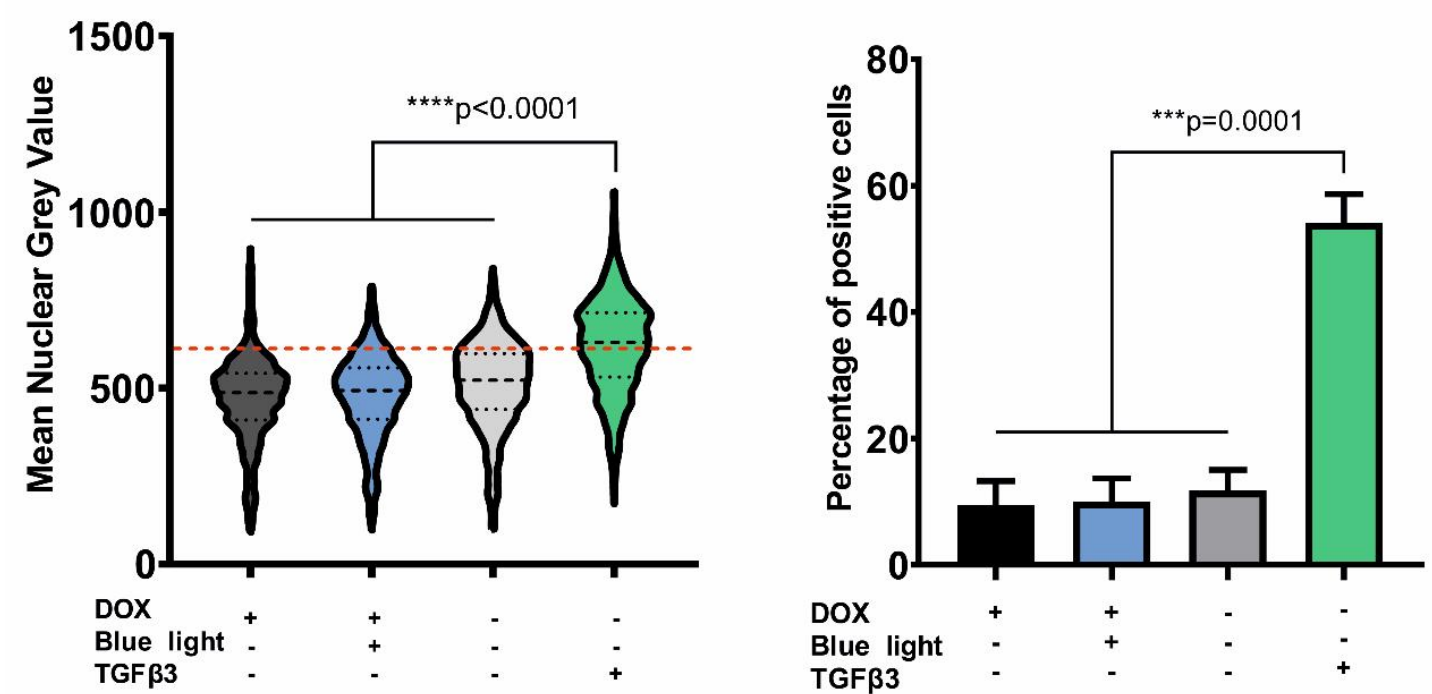

Figure S3. Analysis of SMAD2 nuclear translocation in optoBMP-TC28a2 cells. (A) Representative immunofluorescence images of cells stained for SMAD2 1 hour after initial stimulation. Cells were either stimulated with 15 minutes blue light illumination or kept in the dark after 24 hours doxycycline treatment and serum starvation (LEFT). To act as controls, cells were left untreated with doxycycline during serum starvation and either remain unstimulated or stimulated with 10ng/ml TGFß3 (RIGHT). (B) Single cell quantification of nuclear SMAD2 mean grey value when cells were stimulated with $10 \mathrm{ng} / \mathrm{ml}$ TGF $\beta 3$ or blue light illumination. (C) Percentage of nuclearSMAD2 positive cells when cells were stimulated with $10 \mathrm{ng} / \mathrm{ml}$ TGF $\beta 3$ or blue light illumination calculated from (B). Threshold for positivity calculated through analysis of unstimulated controls.

Data Information: Nuclei were identified with DAPI channel and relative nuclear fluorescence intensity of SMAD2 was measured with ImageJ. Scale bars represent $100 \mu \mathrm{m}$. Quantification performed using three different fields of view per condition across three independent experiments. Violin plots (B) represent all quantified data. Dotted red line indicates mean grey value threshold for positivity determined through analysis of non-stimulated controls. $P$ values were generated using an ordinary one-way ANOVA $\left({ }^{* * *} p<0.0005,{ }^{* * *} p<0.0001\right)$. 


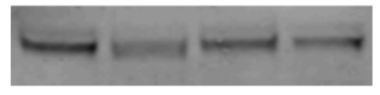

DOX

Blue Light

BMP2

B

PTGS2

HMX3

JUNB
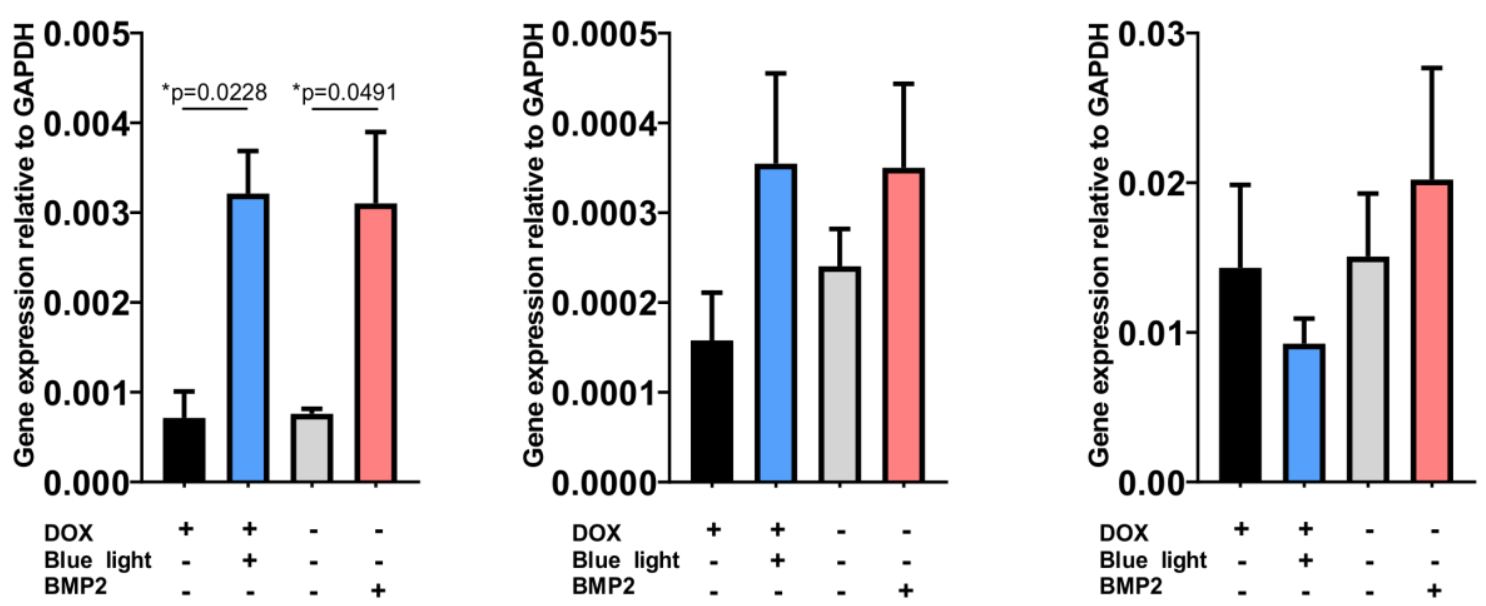

Figure S4. Activation of non-canonical signalling after blue light stimulation in optoBMPTC28a2 cells. (A) Representative western blot for phosphorylated and total ERK1/2 MAPK 2 hours after stimulation with $50 \mathrm{ng} / \mathrm{ml}$ BMP2 or blue light illumination. $\mathrm{N}=3$ runs. (B) Gene expression analyses of known non-canonical BMP signalling pathway targets. Cells were stimulated with blue light illumination or $50 \mathrm{ng} / \mathrm{ml} \mathrm{BMP2}$ and analysed after 4 hours. Gene expression was normalised to GAPDH. $\mathrm{N}$ = four independent experiments.

Data information: Data presented in $B$ represents mean values + SEM. $P$ values were generated using an ordinary one-way ANOVA ( $\left.{ }^{*} \mathrm{p}<0.05\right)$. 
BMPR1A

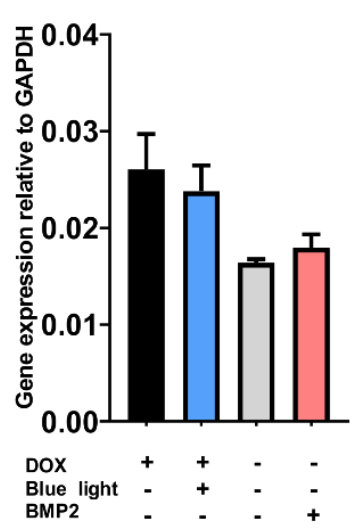

BMPR1B

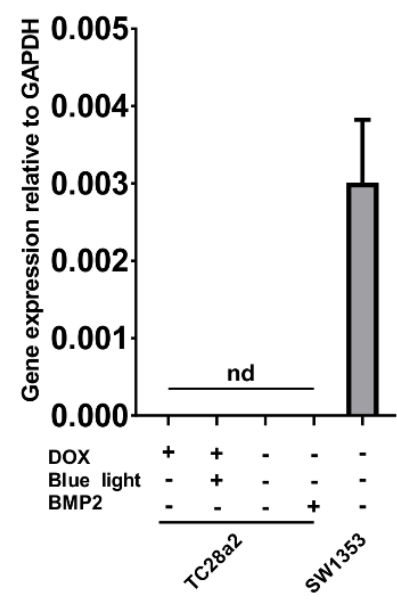

OBMPR1B

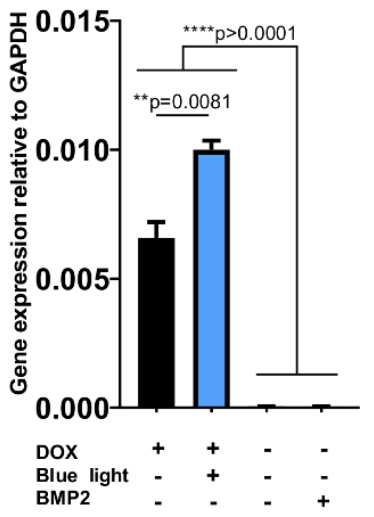

BMPR2

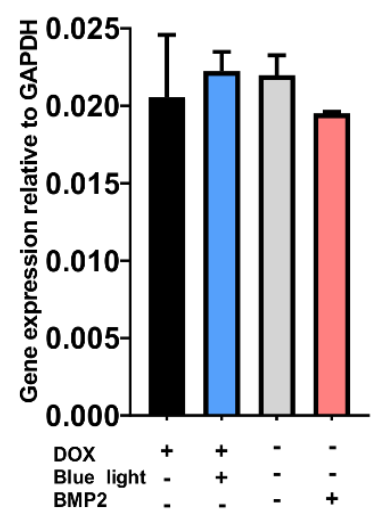

OBMPR2

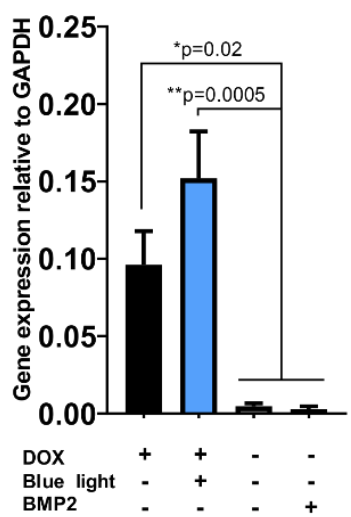

Figure S5 - Gene expression analyses of BMP-like receptors in optoBMP-TC28a2 cells.

Data Information: Native $B M P R 1 B$ and $B M P R 2$ transcript was amplified through primer design targeting exons within the extra-cellular coding regions. BMPR1B transcript was not detected (nd) and primers were validated in an alternative chondrosarcoma SW1353 cell line. Optogenetic receptor transcript was detected through reverse direction primer design targeting the LOV domain coding region. Gene expression was normalised to GAPDH. N = four independent experiments. Bars represent mean values + SEM. $P$ values were generated using an ordinary one-way ANOVA $\left({ }^{*} p<0.05,{ }^{* *} p<0.01,{ }^{* * *} p<0.0001\right)$. 